\title{
DELINEATION OF 3D REFLECTOR GEOMETRIES USING 3D POST STACK DEPTH MIGRATION
}

JEFF RUTLEDGE and ANDY PIEPRZAK Schlumberger/Geco-Prakla, Schlumberger House, Buckingham Gate, Gatwick Airport, West Sussex RH6 ONZ, UK

Exploration targets in structurally complicated areas with strong velocity contrasts require interpretation of seismic data imaged in depth. In areas where conventional processing which includes dip-moveout correction yields CMP-stacked data with good signal strength and continuity, 3-D poststack depth migration offers an attractive solution to the imaging problem. Moreover, migration itself can be used for building and refining velocity-depth models layerby-layer starting from the surface. In such a procedure, coniplete model is constructed by combining interval velocity information with reflector geometries obtained from interpretation of depth migrated seismic horizons. It follows that quality of final migrated result depends strongly on the fidelity of migration algorithm and its implementation. In particular, positioning accuracy, dip response and amplitude preservation characteristics of migration are of importance in addition to issues of turnaround time.

We have implemented a highly-efficient 3-D poststack depth migration scheme on a massively parallel computer without sacrificing accuracy for computational speed. The method is based on an explicit downward continuation in the frequency-space domain allowing for accurate handling of arbitrary lateral and vertical velocity variations. At each depth step, a twodimensional extrapolation operator is created by rotating a one-dimensional explicit operator using the McClellan transform. We differ from the original scheme first presented by Hale in two ways. First, to attain spatially compact operators, we use a Remez type filter design for the one-dimensional filters rather than a design based on least squares. With our implementation, we have achieved higher phase accuracy with minimum amplitude distortions in migrating steep dips up to 75 degrees than previously reported methods. Second, an improved design of the McClellan transform yields near-perfect circular symmetry of the 3-D impulse response, thus correctly positioning steep dips at all azimuths. 
For particularly difficult design situations, where the operators require high fidelity up to a high percentage of the Nyquist wavenumber, the difference between a migration using the Remez and least squares can be striking as given in Figure 1. Figure 1 is an in-line extracted from a 3-D survey. The survey characteristics, trace spacing of $25 \mathrm{~m}$, dominant frequency of $30 \mathrm{~Hz}$, and medium velocities above the salt near $2500 \mathrm{~m} / \mathrm{s}$, means that the design must maintain fidelity up to $84 \%$ of Nyquist wavenumber, even at moderate angles. It can be seen from Figure 1 that the Remez design, gave a better imaging quality, which is especially noticeable on the right-and side of the salt dome, where the dips are nearly 45 degrees. For the same filter length, the Remez design was able to resolve the dips, whereas the least square design had to back off of the maximum angle of resolution to accommodate the difficulty of design. Note that since filter length is the same for this comparison, the migration turnaround time remains the same.

Implementation of the method on a massively parallel computer has reduced turnaround time for migrating 3-D volumes of stacked data from very large 3-D surveys in the order of several milition traces. For example, a 3-D volume stacked data with more than four million traces was migrated down to four $\mathrm{km}$ using a signal bandwidth of 3-70 Hz in ten days.

In our presentation, we shall illustrate the fidelity of our migration method on synthetic datasets designed to emphasize requirements for positioning accuracy, and signal and dip preservation. Real data comparisons will be provided to illustrate the significance of migration operator design in cases of practical importance.

\section{References:}

Hale, D., 1991, 3-D depth migration via McClellan transforms: Geophysics, 56, 1778-1785.

Parks, T., and McClellan, J. 1972, Chebyshev Approximation for Nonrecursive Digital Filters with Linear Phase: IEEE Trans. Circuit Theory, CT-19, 189-194. 

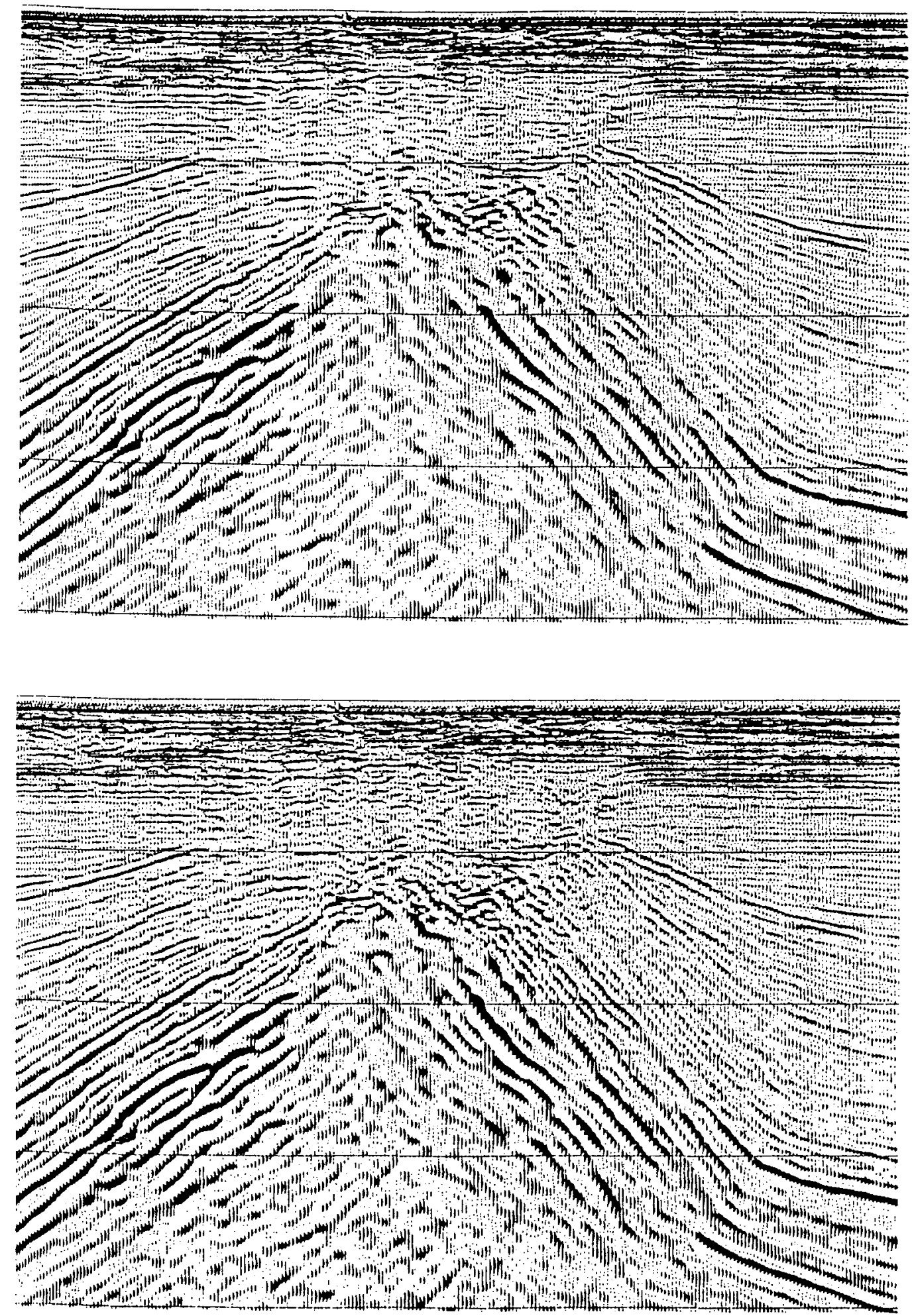

Figure 1. Example section from 3-D depth migration using operators designed by least squares (top) and Remez Exchange Method (bottom) 
